Groups Geom. Dyn. 8 (2014), 225-244

DOI $10.4171 / \mathrm{GGD} / 223$
Groups, Geometry, and Dynamics

(C) European Mathematical Society

\title{
The girth alternative for mapping class groups
}

\author{
Kei Nakamura
}

\begin{abstract}
The girth of a finitely generated group $G$ is defined to be the supremum of the girth of its Cayley graphs. Let $G$ be a finitely generated subgroup of the mapping class group $\operatorname{Mod}_{\Sigma}$, where $\Sigma$ is an orientable closed surface with a finite number of punctures and with a finite number of components. We show that $G$ is either a non-cyclic group with infinite girth or a virtually free-abelian group; these alternatives are mutually exclusive. The proof is based on a simple dynamical criterion for a finitely generated group to have infinite girth, which may be of independent interest.
\end{abstract}

Mathematics Subject Classification (2010). 20E07, 20F38; 05C38, 20F05, 57M07, 57M60.

Keywords. Mapping class groups, girth of Cayley graphs.

\section{Introduction}

Let $\Sigma$ be an orientable closed surface with a finite number of punctures and with a finite number of components. The mapping class group of $\Sigma$, denoted by $\operatorname{Mod}_{\Sigma}$, is the group of isotopy classes of the group $\mathrm{Homeo}^{+}(\Sigma)$ of orientation preserving homeomorphisms of $\Sigma$. Mapping class groups have been studied in complex analysis, low-dimensional topology, and geometric group theory for more than a century.

Mapping class groups share many properties with lattices in linear groups. One analogy between linear groups and mapping class groups can be seen in the following famous dichotomy regarding their subgroups, known as the Tits-alternative for linear groups and mapping class groups respectively.

Theorem ([30]). Let $\mathbb{k}$ be a field, and let $G$ be a finitely generated subgroup of $\mathrm{GL}(n, \mathbb{k})$. Then $G$ either contains a non-abelian free subgroup or is virtually solvable; moreover, these alternatives are mutually exclusive.

Theorem ([12], [20]). Let $\Sigma$ be an orientable closed surface with a finite number of punctures and with a finite number of components, and let $G$ be a finitely generated subgroup of $\operatorname{Mod}_{\Sigma}$. Then $G$ either contains a non-abelian free subgroup or is virtually free-abelian; moreover, these alternatives are mutually exclusive. 
Some refinements and variations of the Tits alternative are known for linear groups and mapping class groups, such as the Margulis-Sorfer theorem on maximal subgroups of linear groups [19] and the analogous result by Ivanov for mapping class groups [12]. This article demonstrates that the structural analogy between these groups can also be witnessed in the girth of a finitely generated subgroup.

Recall that the girth of a graph is the length of the shortest graph cycle, if any, in the graph. The girth of a Cayley graph has been studied extensively, particularly in its relationship to expansion properties; see, for example, [7], [8], [16], [18]. In [27], Schleimer introduced the girth of a finitely generated group $G$, denoted by $\operatorname{girth}(G)$ in this article, to be the supremum of the girth of Cayley graphs of $G$ over all finite generating sets. The qualitative difference between groups with finite girth and groups with infinite girth is evident in observations of Schleimer [27] and the work of Akhmedov [3]. In particular, Akhmedov gave the following girth alternative for linear groups, which shows that the dichotomy between finite girth and infinite girth coincides essentially with the dichotomy in the Tits alternative for linear groups.

Theorem ([3]). Let $\mathbb{k}$ be a field, and let $G$ be a finitely generated subgroup of $\mathrm{GL}(n, \mathbb{k})$. Then $G$ is either a non-cyclic group with infinite girth or a virtually solvable group; moreover, these alternatives are mutually exclusive.

Our result is the following analogous girth alternative for mapping class groups, showing that the dichotomy between subgroups with finite girth and the ones with infinite girth again coincides essentially with the dichotomy in the Tits alternative.

Theorem 1. Let $\Sigma$ be an orientable closed surface with a finite number of punctures and with a finite number of components, and let $G$ be a finitely generated subgroup of $\operatorname{Mod}_{\Sigma}$. Then $G$ is either a non-cyclic group with infinite girth or a virtually free-abelian group; moreover, these alternatives are mutually exclusive.

In the general context of finitely generated groups, the property of having infinite girth is neither stronger nor weaker than the property of containing non-abelian free subgroups; see $\$ 2$ and references therein. Hence, the girth alternative is, a priori, neither stronger nor weaker than the Tits alternative; indeed, Akhmedov recently announced [4] that the girth alternative holds for subgroups of $\mathrm{PL}^{+}(I)$, i.e., the group of orientation-preserving piecewise-linear homeomorphisms of a closed interval, although the Tits alternative fails for subgroups of $\mathrm{PL}^{+}(I)$.

As we shall see, for mapping class groups, the girth alternative is a slightly more intricate manifestation of underlying structural properties that are responsible for the Tits alternative. Our proof of Theorem 1 capitalizes on Proposition 2 below, a criterion for a group to have infinite girth, which generalizes and reformulates the work of Akhmedov [3]; Proposition 2 is quite versatile and can be applied to many classes of groups for which the Tits alternative is known to hold [21]. 
Proposition 2. Let $G$ be a group acting on a set $X$, with a finite generating set $S:=\left\{\gamma_{1}, \ldots, \gamma_{n}\right\}$. Suppose there exist elements $\sigma, \tau \in G$, subsets $U_{\sigma}, U_{\tau} \subset X$, and a point $x \in X$, such that

(1) $x \notin\left(U_{\sigma} \cup U_{\tau}\right) \cup \bigcup_{\varepsilon= \pm 1} \bigcup_{i=1}^{n} \gamma_{i}^{\varepsilon}\left(U_{\sigma} \cup U_{\tau}\right)$,

(2)

$$
\sigma^{k}\left(\{x\} \cup U_{\tau} \cup \bigcup_{\varepsilon= \pm 1} \bigcup_{i=1}^{n} \gamma_{i}^{\varepsilon}\left(U_{\tau}\right)\right) \subset U_{\sigma} \text { for all } k \in \mathbb{Z}-\{0\} \text {, and }
$$

(3) $\tau^{k}\left(\{x\} \cup U_{\sigma} \cup \bigcup_{\varepsilon= \pm 1} \bigcup_{i=1}^{n} \gamma_{i}^{\varepsilon}\left(U_{\sigma}\right)\right) \subset U_{\tau}$ for all $k \in \mathbb{Z}-\{0\}$.

Then $G$ is a non-cyclic group with $\operatorname{girth}(G)=\infty$.

One difficulty in proving Theorem 1 is that Proposition 2 cannot be readily applied to a subgroup $G<\operatorname{Mod}_{\Sigma}$ when $\Sigma$ is disconnected or when $G$ is reducible; see $\S 4$ for the definition of reducible subgroups. Moreover, unlike the Tits alternative, it is not sufficient to prove the statement of Theorem 1 for a finite index subgroup of $G$; it is not known if a group $G$ has infinite girth when $G$ has a finite index subgroup with infinite girth. To work around these issues, we will study the structure of the subgroup $G<\operatorname{Mod}_{\Sigma}$ carefully with Ivanov's theory [13]; some aspects of his theory that were essential in the proof of the Margulis-Soifer theorem for $\operatorname{Mod}_{\Sigma}$, which is much stronger than the Tits alternative, are crucial in our work as well.

For the special case of irreducible subgroups of $\operatorname{Mod}_{\Sigma}$ of a connected surface $\Sigma$, the girth alternative was obtained independently by Yamagata [31]; see $\S 4$ for the definition of an irreducible subgroup. Her result is equivalent to our Proposition 15 in $\S 5$, and her proof is based on a criterion for a group to have infinite girth, somewhat similar to, but weaker than, Proposition 2.

Outline. In $\S 2$, we review some results on the girth of finitely generated groups, and give the proof of Proposition 2, which is a reminiscent of the classical ping-pong argument. In $\S 3$ and $\S 4$, we review the properties of elements and subgroups of $\operatorname{Mod}_{\Sigma}$ from Thurston's theory [29], [6], and Ivanov's theory [13], and collect a few observations that are necessary for the application of Proposition 2 in the proof of Theorem 1. Finally, in §5, we prove Theorem 1.

Conventions. Throughout the article, a surface $\Sigma$ will always be an orientable closed surface with a finite number of punctures and possibly with a finite number of components; for brevity, such a surface will be referred to as a finite-type surface without boundary. We emphasize that, by definition, an element of $\operatorname{Mod}_{\Sigma}$ may permute the punctures and the components of $\Sigma$.

A simple closed curve on $\Sigma$ is said to be peripheral if it bounds a closed disk with one puncture in its interior. A multi-loop on $\Sigma$ is a pairwise disjoint collection of 
simple closed curves on $\Sigma$, and it is said to be essential if each component is neither null-homotopic, peripheral, nor parallel to another component.

Acknowledgements. The author is grateful for numerous invaluable comments and suggestions from multiple referees to improve the exposition. The results in this article appeared as a section in the author's dissertation [21] at University of California, Davis; the author would like to thank Dmitry Fuchs, Misha Kapovich, and his advisor Joel Hass for carefully reading the original exposition of this work and providing insightful comments. Finally, the author would also like to thank Joel Hass, Dave Futer, and Igor Rivin for encouraging him to make this work publicly available in the present format.

\section{Girth of finitely generated groups}

The girth of a graph $\mathcal{E}$, denoted by girth $(\mathcal{E})$, is the combinatorial length of the shortest nontrivial cycle in $\mathcal{E}$ if there is such a cycle, and is set to be infinity if there is no nontrivial cycle in $\mathcal{E}$. Using the girth of Cayley graphs, Schleimer introduced in [27] the notion of the girth of a finitely generated group. Throughout the article, we will always assume that a group is finitely generated.

Definition. Let $G$ be a group. For each generating set $S$ of $G$, let $\mathcal{E}(G, S)$ be the Cayley graph of $G$ with respect to $S$. The girth of the group $G$, denoted by $\operatorname{girth}(G)$, is defined to be $\operatorname{girth}(G):=\sup _{S}\{\operatorname{girth}(\mathscr{E}(G, S))\}$, where the supremum is taken over all finite generating sets $S$ of $G$.

Clearly, every free group has infinite girth, and every finite group has finite girth; it is also easy to see that an abelian group has finite girth unless it is infinite-cyclic. In this section, we discuss criteria for the girth of a group to be finite or infinite.

2.1. Subgroups and quotients. We first collect a few results from [27] and [3] on the relationship between the girth and homomorphisms.

Lemma 3 ([27]). If a group $G$ surjects onto a finite-kernel quotient $G^{\prime}$ with $\operatorname{girth}\left(G^{\prime}\right)<\infty$, then $\operatorname{girth}(G)<\infty$.

Lemma 4 ([27]). If a group $G$ contains a finite-index subgroup $G^{\prime \prime}<G$ with $\operatorname{girth}\left(G^{\prime \prime}\right)<\infty$, then $\operatorname{girth}(G)<\infty$.

Proposition 5 ([3]). If a group $G$ surjects onto a non-cyclic quotient $G^{\prime}$ with $\operatorname{girth}\left(G^{\prime}\right)=\infty$, then $\operatorname{girth}(G)=\infty$.

It should be noted that there exists a group with finite girth, containing a subgroup with infinite girth; more specifically, Akhmedov [2], §4, observed that Olshanskii's 
result [25], §34-35, can be reformulated to produce a group with finite girth, containing an infinite-index non-abelian free subgroup. Akhmedov has also shown that a group has infinite girth if it contains a finite-index non-abelian free subgroup [3], §2. However, it is not known in general if a group has infinite girth when a finite-index subgroup has infinite girth. In the proof of Theorem 1, instead of studying the girth of finite-index subgroups, we look for a surjection of the given group onto another group with infinite girth and apply Proposition 5.

2.2. Criterion for finite girth. Generalizing the fact that every abelian group $G \nsubseteq$ $\mathbb{Z}$ has finite girth, Schleimer obtained a useful criterion for a group to have finite girth [27]. Recall that a group $G$ is said to satisfy a law if there is a word $w\left(x_{1}, \ldots, x_{n}\right)$ on $n$ letters such that $w\left(\gamma_{1}, \ldots, \gamma_{n}\right)=1$ in $G$ for any $\gamma_{1}, \ldots, \gamma_{n} \in G$.

Theorem 6 ([27]). If a group $G$ satisfies a law and $G \not \mathbb{Z}$, then $\operatorname{girth}(G)<\infty$.

Since every solvable group satisfies a law, Theorem 6 and Proposition 4 together yield the following corollary which is relevant to the girth alternative.

Corollary 7. If a group $G$ is virtually solvable and $G \not \mathbb{Z}$, then $\operatorname{girth}(G)<\infty$.

More generally, every uniformly amenable group satisfies a law [14]. There are also non-amenable groups satisfying some law, such as Tarski Monster groups [23], [24], many free Burnside groups [1], and Olshanskii-Sapir groups [26]. By Theorem 6, these groups all have finite girth. We note that there also exists a group with finite girth, satisfying no law; as mentioned in $\S 2.1$, there exists a group with finite girth, containing a non-abelian free subgroup, see [2], §4, and [25], §34-35, and such a group clearly satisfies no law.

2.3. Criterion for infinite girth. The proof of the Tits alternative for linear groups and mapping class groups, as well as for other classes of groups, use variations of the so-called ping-pong lemma to construct a non-abelian free subgroup; the classical lemma goes back to the work of Blaschke, Klein, Schottky, and Poincaré; see, for example, [15]. The following formulation was given in [30].

Proposition 8 ([30]). Let $G$ be a group acting on a set $X$. Suppose there exist elements $\sigma, \tau \in G$, subsets $U_{\sigma}, U_{\tau} \subset X$, and a point $x \in X$, such that

(1) $x \notin U_{\sigma} \cup U_{\tau}$,

(2) $\sigma^{k}\left(\{x\} \cup U_{\tau}\right) \subset U_{\sigma}$ for all $k \in \mathbb{Z}-\{0\}$, and

(3) $\tau^{k}\left(\{x\} \cup U_{\sigma}\right) \subset U_{\tau}$ for all $k \in \mathbb{Z}-\{0\}$.

Then $\langle\sigma, \tau\rangle$ is a non-abelian free subgroup of $G$. 
Proof. Observe inductively that any nontrivial reduced word in $\sigma^{ \pm 1}$ and $\tau^{ \pm 1}$ takes $x \in X-\left(U_{\sigma} \cup U_{\tau}\right)$ into $U_{\sigma} \cup U_{\tau}$ via the action of $\langle\sigma, \tau\rangle$, showing that the word cannot represent the identity element of $G$. This is the classical ping-pong argument.

In the study of the girth of groups, it is instrumental to have a criterion for a group to have infinite girth. Generalizing and reformulating the ideas appearing in the work of Akhmedov [3], we give the following criterion, as stated in $\S 1$.

Proposition 2. Let $G$ be a group acting on a set $X$, with a finite generating set $S:=\left\{\gamma_{1}, \ldots, \gamma_{n}\right\}$. Suppose there exist elements $\sigma, \tau \in G$, subsets $U_{\sigma}, U_{\tau} \subset X$, and a point $x \in X$, such that

(1) $x \notin\left(U_{\sigma} \cup U_{\tau}\right) \cup \bigcup_{\varepsilon= \pm 1} \bigcup_{i=1}^{n} \gamma_{i}^{\varepsilon}\left(U_{\sigma} \cup U_{\tau}\right)$,

(2) $\sigma^{k}\left(\{x\} \cup U_{\tau} \cup \bigcup_{\varepsilon= \pm 1} \bigcup_{i=1}^{n} \gamma_{i}^{\varepsilon}\left(U_{\tau}\right)\right) \subset U_{\sigma}$ for all $k \in \mathbb{Z}-\{0\}$, and

(3) $\tau^{k}\left(\{x\} \cup U_{\sigma} \cup \bigcup_{\varepsilon= \pm 1} \bigcup_{i=1}^{n} \gamma_{i}^{\varepsilon}\left(U_{\sigma}\right)\right) \subset U_{\tau}$ for all $k \in \mathbb{Z}-\{0\}$.

Then $G$ is a non-cyclic group with $\operatorname{girth}(G)=\infty$.

Since $\sigma, \tau, U_{\sigma}, U_{\tau}$, and $x \in X$ in Proposition 2 satisfy the conditions in Proposition 8, we see that $\sigma$ and $\tau$ generate a non-abelian free subgroup $\langle\sigma, \tau\rangle<G$. Proposition 2 applies to many groups which are known to contain non-abelian free subgroup by some version of the ping-pong argument; in the present work, we focus on the application of Proposition 2 to mapping class groups. The criterion can also be applied to non-elementary convergence groups, subgroups of $\operatorname{Out}\left(F_{n}\right)$ containing strongly irreducible elements, many CAT $(0)$ spaces, and some groups acting on trees; some results on the girth of these groups are collected in [21].

Although every group that satisfies Proposition 2 contains a non-abelian free subgroup, the property of having infinite girth and the property of containing a nonabelian free subgroup are generally independent of each other. As we have noted in $\S 2.1$, there exists a group with finite girth, containing a non-abelian free subgroup, [2], §4, [25], §34-35. On the other hand, there also exists a group with infinite girth, containing no non-abelian free subgroups [2], §2. Thompson's group $F$ is another example of a group with infinite girth [9], [5], containing no non-abelian free subgroup [10].

Proof of Proposition 2. Let $M$ be a positive integer, and we aim to find a new generating set $\hat{S}$ for $G$ such that $\operatorname{girth}(\mathscr{G}(G, \hat{S})) \geq M$. Let $P=\left\{p_{1}, \ldots, p_{n}\right\}$ be a set of positive integers such that $p_{i}>M$ for all $i$ and $\left|p_{i}-p_{j}\right|>M$ for all distinct $i, j$. Let $\hat{\gamma}_{i}:=\sigma^{p_{i}} \gamma_{i} \tau^{-p_{i}}$ for each $i$. The set $\hat{S}:=\left\{\sigma, \tau, \hat{\gamma}_{1}, \ldots, \hat{\gamma}_{n}\right\}$ clearly generates $G$. 
Let $w$ be a nontrivial reduced word in $\hat{S} \cup \hat{S}^{-1}$ of length less than $M$ with respect to $\hat{S} \cup \hat{S}^{-1}$. We can write $w$ as

$$
w=u_{1} \hat{\gamma}_{i_{1}}^{\varepsilon_{1}} u_{2} \hat{\gamma}_{i_{2}}^{\varepsilon_{2}} \ldots \hat{\gamma}_{i_{s}}^{\varepsilon_{s}} u_{s+1}
$$

where $\varepsilon_{\ell} \in\{ \pm 1\}$ and the subword $u_{\ell}=u_{\ell}(\sigma, \tau)$ is a (possibly empty) reduced word in $\left\{\sigma^{ \pm 1}, \tau^{ \pm 1}\right\}$. If $u_{\ell}$ is an empty word and $i_{\ell-1}=i_{\ell}$ for some $\ell$, we must have $\varepsilon_{\ell-1}=\varepsilon_{\ell}$. For otherwise, a cancellation occurs and contradicts the assumption that $w$ is a reduced word in $\hat{S} \cup \hat{S}^{-1}$.

Now, regarded as an element of $G, w$ can be expressed as

$$
\begin{aligned}
w & =u_{1} \hat{\gamma}_{i_{1}}^{\varepsilon_{1}} u_{2} \hat{\gamma}_{i_{2}}^{\varepsilon_{2}} \ldots \hat{\gamma}_{i_{s}}^{\varepsilon_{s}} u_{s+1} \\
& =v_{1} \gamma_{i_{1}}^{\varepsilon_{1}} v_{2} \gamma_{i_{2}}^{\varepsilon_{2}} \ldots \gamma_{i_{s}}^{\varepsilon_{s}} v_{s+1}
\end{aligned}
$$

where $v_{\ell}=v_{\ell}(\sigma, \tau)$ is a reduced word in $\left\{\sigma^{ \pm 1}, \tau^{ \pm 1}\right\}$ for $\beta_{\ell-1} u_{\ell} \alpha_{\ell}$, with convention $\alpha_{s+1}=\beta_{0}=1$, and

$$
\alpha_{\ell}=\left\{\begin{array}{ll}
\sigma^{p_{i_{\ell}}} & \text { if } \varepsilon_{\ell}=+1, \\
\tau^{p_{i_{\ell}}} & \text { if } \varepsilon_{\ell}=-1,
\end{array} \quad \beta_{\ell}= \begin{cases}\tau^{-p_{i_{\ell}}} & \text { if } \varepsilon_{\ell}=+1, \\
\sigma^{-p_{i_{\ell}}} & \text { if } \varepsilon_{\ell}=-1 .\end{cases}\right.
$$

The idea of the proof is to apply the ping-pong argument to $w$ to show that $w$ cannot represent the identity element of $G$. Provided with suitable initial points in $X$, the ping-pong argument applies easily to the strings $v_{\ell}$. What we need to show is that we can pass each $\gamma_{i_{\ell}}^{\varepsilon_{\ell}}$ in the ping-pong argument; in other words, we need to check that $\gamma_{i_{\ell}}^{\varepsilon_{\ell}}$ takes the terminal point from the ping-pong rally $v_{\ell+1}$ to a suitable initial point for the ping-pong rally $v_{\ell}$. We will see that our choice of $p_{i}$ prevents excessive cancellations, and we can indeed pass each $\gamma_{i_{\ell}}^{\varepsilon_{\ell}}$ under the conditions (2) and (3) in the statement of the proposition.

Claim 1. For each $\ell, v_{\ell}$ is not an empty word. If $\varepsilon_{\ell}=+1$, then the last letter of $v_{\ell}$ is $\sigma^{ \pm 1}$ and the first letter of $v_{\ell+1}$ is $\tau^{ \pm 1}$. If $\varepsilon_{\ell}=-1$, then the last letter of $v_{\ell}$ is $\tau^{ \pm 1}$ and the first letter of $v_{\ell+1}$ is $\sigma^{ \pm 1}$.

Proof of Claim 1. Let us show that, if $\varepsilon_{\ell}=+1$, then $v_{\ell}$ is a non-empty word ending with $\sigma^{ \pm 1}$. Since $\varepsilon_{\ell}=+1$, we have $\alpha_{\ell}=\sigma^{p_{i}}$ and $\beta_{\ell}=\tau^{-p_{i_{\ell}}}$. There are three cases to consider: (i) $u_{\ell}$ is an empty word; (ii) the last letter of $u_{\ell}$ is $\tau^{ \pm 1}$; or (iii) the last letter of $u_{\ell}$ is $\sigma^{ \pm 1}$.

Case (i): If $u_{\ell}$ is empty, then $v_{\ell}$ is the reduced word for $\beta_{\ell-1} \alpha_{\ell}$, and hence

$$
v_{\ell}= \begin{cases}\sigma^{p_{i_{\ell}}} & \text { if } \ell=1, \\ \tau^{-p_{i_{\ell-1}} \sigma^{p_{i_{\ell}}}} & \text { if } \ell \neq 1 \text { and } \varepsilon_{\ell-1}=+1, \\ \sigma^{-p_{i_{\ell-1}}+p_{i_{\ell}}} & \text { if } \ell \neq 1 \text { and } \varepsilon_{\ell-1}=-1 .\end{cases}
$$

In the last subcase, since $\varepsilon_{\ell}=+1 \neq-1=\varepsilon_{\ell-1}$, we must have $i_{\ell} \neq i_{\ell-1}$ as noted before. Thus, we must have $\left|p_{i_{\ell}}-p_{i_{\ell-1}}\right|>M$, and it follows that $v_{\ell}$ is a nontrivial power of $\sigma$. In all subcases, $v_{\ell}$ is indeed a non-empty word ending with $\sigma^{ \pm 1}$. 
Case (ii): If the last letter of $u_{\ell}$ is $\tau^{ \pm 1}$, then there is no cancellation between $u_{\ell}$ and $\alpha_{\ell}=\sigma^{p_{i}}$ as a word in $\left\{\sigma^{ \pm 1}, \tau^{ \pm 1}\right\}$. Hence, $v_{\ell}$ is again a non-empty word ending with $\sigma^{ \pm 1}$.

Case (iii): Finally, suppose the last letter of $u_{\ell}$ is $\sigma^{ \pm 1}$. If $u_{\ell}$ is not a power of $\sigma$, then $u_{\ell}=\ldots \tau^{q} \sigma^{p}$ for some $q$ and $p$. So, $u_{\ell} \alpha_{\ell}=\ldots \tau^{q} \sigma^{p+p_{i}}$. Note that we must have $|p|<M$. For otherwise, the length of $u_{\ell}$ as a word in $\hat{S} \cup \hat{S}^{-1}$, and thus the length of $w$ as a word in $\hat{S} \cup \hat{S}^{-1}$, is at least $M$; this contradicts with the assumption on the length of $w$. Now, $|p|<M$ and $p_{i_{\ell}}>M$ together imply $p+p_{i_{\ell}} \neq 0$. Thus, the last letter of $v_{\ell}$ must be $\sigma^{ \pm 1}$. If $u_{\ell}$ is a power of $\sigma$, say $u_{\ell}=\sigma^{p}$, then

$$
v_{\ell}= \begin{cases}\sigma^{p+p_{i_{\ell}}} & \text { if } \ell=1, \\ \tau^{-p_{i_{\ell-1}}} \sigma^{p+p_{i_{\ell}}} & \text { if } \ell \neq 1 \text { and } \varepsilon_{\ell-1}=+1, \\ \sigma^{-p_{i_{\ell-1}}+p+p_{i_{\ell}}} & \text { if } \ell \neq 1 \text { and } \varepsilon_{\ell-1}=-1 .\end{cases}
$$

In the first two subcases, $v_{\ell}$ ends with a nontrivial power of $\sigma$, because $|p|<M$ and $p_{i_{\ell}}>M$ imply $p+p_{i_{\ell}} \neq 0$. In the third subcase, we must have $i_{\ell} \neq i_{\ell-1}$, and hence $\left|p_{i_{\ell}}-p_{i_{\ell-1}}\right|>M$. It now follows from $|p|<M$ that $-p_{i_{\ell-1}}+p+p_{i_{\ell}} \neq 0$, and $v_{\ell}$ is again a nontrivial power of $\sigma$. Thus, in all subcases, $v_{\ell}$ is again a non-empty word ending with $\sigma^{ \pm 1}$ as desired.

This concludes the proof that, if $\varepsilon_{\ell}=+1$, then $v_{\ell}$ is a non-empty word ending with $\sigma^{ \pm 1}$. The analogous arguments show that, if $\varepsilon_{\ell}=+1$, then $v_{\ell+1}$ is a non-empty word beginning with $\tau^{ \pm 1}$. The symmetric arguments show that, if $\varepsilon_{\ell}=-1$, then $v_{\ell}$ is a nonempty word ending with $\tau^{ \pm 1}$ and $v_{\ell+1}$ is a non-empty word beginning with $\sigma^{ \pm 1}$.

Claim 2. If the last letter of $v_{s+1}$ is $\sigma^{ \pm 1}$ and $y \in\{x\} \cup U_{\tau} \cup \bigcup_{\varepsilon= \pm 1} \bigcup_{i=1}^{n} \gamma_{i}^{\varepsilon}\left(U_{\tau}\right)$, or if the last letter of $v_{s+1}$ is $\tau^{ \pm 1}$ and $y \in\{x\} \cup U_{\sigma} \cup \bigcup_{\varepsilon= \pm 1} \bigcup_{i=1}^{n} \gamma_{i}^{\varepsilon}\left(U_{\sigma}\right)$, then $w(y) \in U_{\sigma} \cup U_{\tau}$.

Proof of Claim 2. We will prove the claim by induction on $s$. If $s=0, w=v_{1}$ is merely a reduced word in $\sigma^{ \pm 1}$ and $\tau^{ \pm 1}$. In this case, $w(y) \in U_{\sigma} \cup U_{\tau}$ follows from the classical ping-pong argument as in the proof of the Free Subgroup Criterion.

Now, as the induction hypothesis, suppose that the claim is true for $s-1 \geq 0$, and let $w=v_{1} \gamma_{i_{1}}^{\varepsilon_{1}} v_{2} \gamma_{i_{2}}^{\varepsilon_{2}} \ldots \gamma_{i_{s}}^{\varepsilon_{s}} v_{s+1}$. Suppose $\varepsilon_{s}=+1$ for now, so that the first letter of $v_{s+1}$ is $\tau^{ \pm 1}$ by Claim 1. Then we have $v_{s+1}(y) \in U_{\tau}$ by the classical ping-pong argument, and we obtain $y^{\prime}:=\gamma_{i_{s}} v_{s+1}(y) \in \gamma_{i_{s}}\left(U_{\tau}\right)$. Now, also by Claim 1, the last letter of $v_{s}$ is $\sigma^{ \pm 1}$. Thus, applying the induction hypothesis to $w^{\prime}:=v_{1} \gamma_{i_{1}}^{\varepsilon_{1}} v_{2} \gamma_{i_{2}}^{\varepsilon_{2}} \ldots \gamma_{i_{s-1}}^{\varepsilon_{s-1}} v_{s}$ and $y^{\prime}$, we see that $w(y)=w^{\prime}\left(y^{\prime}\right) \in U_{\sigma} \cup U_{\tau}$. The $\varepsilon_{s}=-1$ case is analogous.

Since $x \notin U_{\sigma} \cup U_{\tau}$ by the assumption and $w(x) \in U_{\sigma} \cup U_{\tau}$ by Claim 2, it follows that $w$ cannot represent the identity element in $G$. Namely, any non-empty word in $\hat{S}$ that represents the identity element of $G$ must be of length at least $M$ with respect to $\hat{S}$. Hence, $\operatorname{girth}(G) \geq \operatorname{girth}(\mathscr{E}(G, \hat{S})) \geq M$. 


\section{Elements of mapping class groups}

We review some aspects of the Nielsen-Thurston classification of elements of $\operatorname{Mod}_{\Sigma}$, for an orientable finite-type surface $\Sigma$ with $\partial \Sigma=\varnothing$. An isotopy class $\mathcal{A}$ of an essential multi-loop on $\Sigma$ is a reduction system for $\sigma \in \operatorname{Mod}_{\Sigma}$ if $\sigma$ fixes $\mathcal{A}$. An element in $\operatorname{Mod}_{\Sigma}$ is reducible if it admits a non-empty reduction system $\mathcal{A}$, and is irreducible otherwise; it is periodic if its order is finite, and is aperiodic otherwise. There are three mutually exclusive types of elements in $\operatorname{Mod}_{\Sigma}$ : (i) periodic elements, (ii) aperiodic irreducible elements, and (iii) aperiodic reducible elements.

This trichotomy was studied by Nielsen for tori [22], and by Thurston more generally for other surfaces [29]. The theory was further developed by Birman, Lubotzky, and McCarthy [6], [20], and by Ivanov [12], [13], Chap. 2-8, leading to the proof of the Tits alternative for $\operatorname{Mod}_{\Sigma}$. For the rest of this section, we assume that each component of $\Sigma$ has negative Euler characteristic.

3.1. Canonical reduction of reducible elements. Suppose that $\mathcal{A}$ is a reduction system for an element $\sigma \in \operatorname{Mod}_{\Sigma}$, and let $\Sigma_{\mathcal{A}}:=\Sigma-A$ where $A$ is a multi-loop representing $\mathcal{A}$; note that $\Sigma_{\mathscr{A}}$ is again an orientable finite-type surface with $\partial \Sigma_{\mathscr{A}}=\varnothing$, and $\sigma$ induces a mapping class $\rho_{\mathcal{A}}(\sigma) \in \operatorname{Mod}_{\Sigma_{\mathcal{A}}}$, called the reduction of $\sigma$ along $\mathcal{A}$. We say that an element of $\operatorname{Mod}_{\Sigma}$ is neat if it takes each component of $\Sigma$ to itself and each puncture of $\Sigma$ to itself; adopting the terminology from [6], we say that an element $\sigma \in \operatorname{Mod}_{\Sigma}$ is adequately reduced if there exists a neat nontrivial power $\sigma^{m}$ whose restriction to each component of $\Sigma$ is either (i) trivial or (ii) aperiodic and irreducible. A reduction system $\mathcal{A}$ of an element $\sigma \in \operatorname{Mod}_{\Sigma}$ is said to be an adequate reduction system if the reduction $\rho_{\mathcal{A}}(\sigma)$ is adequately reduced.

By Thurston's classification theorem [29], Thm. 4, every element $\sigma \in \operatorname{Mod}_{\Sigma}$ is either an adequately reduced element or an aperiodic reducible element with nonempty adequate reduction systems. For an aperiodic reducible element $\sigma$, there is a canonical choice of a non-empty adequate reduction system, [6], Thm. C, [13], §7.4, $\$ 7.11$; it is indeed the unique minimal adequate reduction system for the element $\sigma$. We call such a system the canonical reduction system for $\sigma$, and denote it by $\ell_{\sigma}$, or simply by $\ell$ if the reference to $\sigma$ is clear from the context.

3.2. Adequately reduced elements. We recall the description of adequately reduced elements based on Thurston's theory. In particular, we review the dynamical properties of aperiodic irreducible elements, acting on the spaces of projective measured foliations on a surface.

Connected surfaces. Suppose for now that $\Sigma$ is connected, and write $g(\Sigma)$ and $n(\Sigma)$ for the genus and the number of punctures of $\Sigma$. It follows from the discussion in $\$ 3.1$ that an element $\sigma \in \operatorname{Mod}_{\Sigma}$ is adequately reduced if and only if it is (i) periodic or (ii) aperiodic and irreducible. We summarize Thurston's characterization [29] of aperiodic irreducible elements; the details can be found in [11], Exp. 8-9. 
Thurston introduced the space $\mathcal{P} \mathcal{M} \mathcal{F}(\Sigma)$ of projective measured foliations on $\Sigma$, homeomorphic to a sphere of dimension $6 g(\Sigma)+2 n(\Sigma)-7$. The mapping class

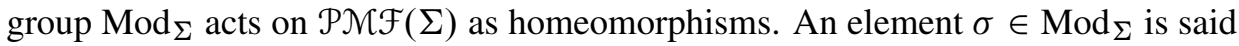
to be pseudo-Anosov if the fixed-point set $\operatorname{Fix}(\sigma) \subset \mathcal{P} \mathcal{M} \mathcal{F}(\Sigma)$ of the action of $\sigma$ on $\mathcal{P} \mathcal{M} \mathcal{F}(\Sigma)$ consists of precisely two points in $\mathcal{P} \mathcal{M} \mathcal{F}(\Sigma)$. An essential property of a pseudo-Anosov element $\sigma$ is that its action on $\mathcal{P \mathcal { M }} \mathcal{F}(\Sigma)$ exhibits the north-south dynamics with one of the fixed points as an attractor, denoted by $\mathcal{F}_{\sigma}^{+}$, and the other as a repeller, denoted by $\mathcal{F}_{\sigma}^{-}$; more precisely, for any pair of disjoint neighborhoods $U_{\sigma}^{+}$of $\mathcal{F}_{\sigma}^{+}$and $U_{\sigma}^{-}$of $\mathcal{F}_{\sigma}^{-}$, we have $\sigma^{ \pm N}\left(\mathcal{P \mathcal { M }} \mathcal{F}(\Sigma)-U_{\sigma}^{\mp}\right) \subset U_{\sigma}^{ \pm}$respectively for all sufficiently large $N$. It turns out that aperiodic irreducible elements of $\operatorname{Mod}_{\Sigma}$ are precisely pseudo-Anosov ones [29], Thm. 4. If $\sigma, \tau \in \operatorname{Mod}_{\Sigma}$ are pseudo-Anosov elements, then $\operatorname{Fix}(\sigma)=\operatorname{Fix}(\tau)$ or $\operatorname{Fix}(\sigma) \cap \operatorname{Fix}(\tau)=\varnothing$ in $\mathcal{P} \mathcal{M} \mathcal{F}(\Sigma)$ [13], §5.11; we say that $\sigma$ and $\tau$ are independent if $\operatorname{Fix}(\sigma) \cap \operatorname{Fix}(\tau)=\varnothing$.

Disconnected surfaces. Let us now allow $\Sigma=\bigsqcup_{i} \Sigma_{i}$ to be disconnected, where $\Sigma_{i}$ denotes each component. With some care, the space $\mathcal{P} \mathcal{M} \mathcal{F}(\Sigma)$ of projective measured foliations on $\Sigma$ can be defined as the join of $\mathcal{P \mathcal { M }} \mathcal{F}\left(\Sigma_{i}\right)$, [13], [20]. However, as in the work of Ivanov [13], Chap. 6, it is sufficient and more convenient for our purposes to consider the space

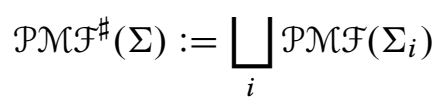

and the action of $\operatorname{Mod}_{\Sigma}$ on it as homeomorphisms. A neat element in $\operatorname{Mod}_{\Sigma}$ takes each component $\mathcal{P} \mathcal{M} \mathcal{F}\left(\Sigma_{i}\right) \subset \mathcal{P} \mathcal{M} \mathcal{F}^{\sharp}(\Sigma)$ to itself under this action.

An element of $\operatorname{Mod}_{\Sigma}$ is said to be pseudo-Anosov if there exists a neat power $\sigma^{m}$ whose restriction to each component $\Sigma_{i}$ is a pseudo-Anosov element in $\operatorname{Mod}_{\Sigma_{i}}$.

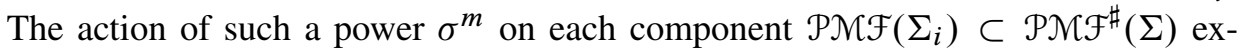
hibits the north-south dynamics with respect to the fixed points $\mathcal{F}_{\sigma^{m} \mid \Sigma_{i}}^{ \pm} \in \mathcal{P} \mathcal{M} \mathcal{F}\left(\Sigma_{i}\right)$. Moreover, $\sigma$ fixes $\bigcup_{i} \mathcal{F}_{\sigma^{m} \mid \Sigma_{i}}^{+}$and $\bigcup_{i} \mathcal{F}_{\sigma^{m} \mid \Sigma_{i}}^{-}$setwise, respectively, and the action of $\sigma$ on $\mathcal{P} \mathcal{M} \mathcal{F}^{\sharp}(\Sigma)$ exhibits the north-south dynamics with respect to these sets; more precisely, for any pair of disjoint neighborhoods $U_{\sigma}^{+}$of $\bigcup_{i} \mathcal{F}_{\sigma^{m} \mid \Sigma_{i}}^{+}$and $U_{\sigma}^{-}$of $\bigcup_{i} \mathcal{F}_{\sigma^{m} \mid \Sigma_{i}}^{-}$, we have $\sigma^{ \pm N}\left(\mathcal{P \mathcal { M F }}^{\sharp}(\Sigma)-U_{\sigma}^{\mp}\right) \subset U_{\sigma}^{ \pm}$for all sufficiently large $N$. If $\sigma, \tau \in \operatorname{Mod}_{\Sigma}$ are pseudo-Anosov elements, then for each $i, \operatorname{Fix}(\sigma) \cap \mathcal{P} \mathcal{M} \mathcal{F}\left(\Sigma_{i}\right)=$

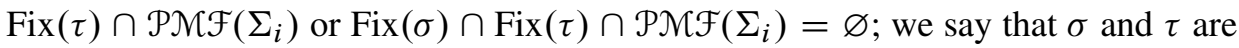
independent if $\operatorname{Fix}(\sigma) \cap \operatorname{Fix}(\tau)=\varnothing$, i.e., $\operatorname{Fix}(\sigma) \cap \operatorname{Fix}(\tau) \cap \mathcal{P \mathcal { M }} \mathcal{F}\left(\Sigma_{i}\right)=\varnothing$ for all $i$.

Recall that an element $\sigma \in \operatorname{Mod}_{\Sigma}$ is adequately reduced if there exists a neat nontrivial power $\sigma^{m}$ whose restriction to each component $\Sigma_{i}$ is either (i) trivial or (ii) aperiodic and irreducible. Clearly, $\sigma$ is periodic if the restriction of $\sigma^{m}$ to each $\Sigma_{i}$ is trivial; also, $\sigma$ is pseudo-Anosov if the restriction of $\sigma^{m}$ to each $\Sigma_{i}$ is aperiodic and irreducible. Generally, an adequately reduced element may be neither periodic nor pseudo-Anosov; the restrictions of $\sigma^{m}$ to some components can be 
trivial while the restrictions of $\sigma^{m}$ to other components are aperiodic and irreducible.

\section{Subgroups of mapping class groups}

We review some aspects of Ivanov's classification [13], Chap. 5-9, of subgroups of $\operatorname{Mod}_{\Sigma}$, for an orientable finite-type surface $\Sigma$ with $\partial \Sigma=\varnothing$. An isotopy class $\mathcal{A}$ of an essential multi-loop is a reduction system for $G<\operatorname{Mod}_{\Sigma}$ if it is a reduction system for every element $\sigma \in G$, i.e., if every element $\sigma \in G$ fixes $\mathcal{A}$. A subgroup $G$ is reducible if it admits a non-empty reduction system $\mathcal{A}$, and is irreducible otherwise. There are three mutually exclusive types of subgroups in $\operatorname{Mod}_{\Sigma}$ : (i) finite subgroups, (ii) infinite irreducible subgroups, and (iii) infinite reducible subgroups. This trichotomy parallels and generalizes the one appearing in the Nielsen-Thurston classification; an element $\sigma \in \operatorname{Mod}_{\Sigma}$ is (i) periodic, (ii) aperiodic and irreducible, or (iii) aperiodic and reducible, if and only if the cyclic subgroup $\langle\sigma\rangle<\operatorname{Mod}_{\Sigma}$ is (i) finite, (ii) infinite and irreducible, or (iii) infinite and reducible, respectively.

Ivanov's classification theory for subgroups of $\operatorname{Mod}_{\Sigma}$, strictly speaking, is much deeper than what is required in the proof of the Tits alternative for $\operatorname{Mod}_{\Sigma}$. However, it played an essential role in his proof of stronger theorems such as the analogue of Margulis-Soifer theorem [12], [13]. In our proof of the girth alternative, we will also need to utilize Ivanov's classification theory. For the rest of this section, we assume that each component of $\Sigma$ has negative Euler characteristic.

4.1. Canonical reduction of reducible subgroups. A reduction of a subgroup of $\operatorname{Mod}_{\Sigma}$ can be defined in a manner analogous to the reduction of an element of $\operatorname{Mod}_{\Sigma}$. If $\mathcal{A}$ is a reduction system for $G<\operatorname{Mod}_{\Sigma}$, then the reduction $\rho_{\mathcal{A}}(\sigma) \in \operatorname{Mod}_{\Sigma_{\mathcal{A}}}$ is well defined for each $\sigma \in G$, where $\Sigma_{\mathcal{A}}$ is defined as in $\$ 3.1$. The assignment $\sigma \mapsto \rho_{\mathcal{A}}(\sigma)$ indeed defines the reduction homomorphism

$$
\rho_{\mathcal{A}}: G \rightarrow \operatorname{Mod}_{\Sigma_{\mathcal{A}}}
$$

whose kernel is a free-abelian group generated by Dehn twists along some components of $\mathcal{A}$; the image $\rho_{\mathcal{A}}(G)$ is called the reduction of $G$ along $\mathcal{A}$. We say a subgroup $G<\operatorname{Mod}_{\Sigma}$ is neat if it consists of neat elements; pushing the analogy further, we say that a subgroup $G<\operatorname{Mod}_{\Sigma}$ is adequately reduced if there exists a neat finite-index normal subgroup $G^{\prime} \triangleleft G$ whose restriction to each component of $\Sigma$ is either (i) trivial or (ii) infinite and irreducible. If $G$ is an adequately reduced group with a neat finite index subgroup $G^{\prime} \triangleleft G$ as in the definition, then, for any finite-index normal subgroup $G^{\prime \prime} \triangleleft G$, a neat finite-index subgroup $G^{\prime} \cap G^{\prime \prime} \triangleleft G^{\prime \prime}$ guarantees that $G^{\prime \prime}$ is also adequately reduced.

Every subgroup $G<\operatorname{Mod}_{\Sigma}$ indeed contains a neat finite-index normal subgroup. In practice, we almost always consider a particular family of such subgroups. For 
each integer $m \geq 3$, we consider the natural homomorphisms

$$
\operatorname{Mod}_{\Sigma} \rightarrow \operatorname{Aut}\left(H_{1}(\Sigma ; \mathbb{Z})\right) \rightarrow \operatorname{Aut}\left(H_{1}(\Sigma ; \mathbb{Z} / m \mathbb{Z})\right)
$$

and let $\operatorname{Mod}_{\Sigma}(m)$ be the kernel of this composition of homomorphisms. $\operatorname{Mod}_{\Sigma}(m)$ is clearly a finite-index normal subgroup of $\operatorname{Mod}_{\Sigma}$, and a classical theorem of Serre [28] says that $\operatorname{Mod}_{\Sigma}(m)$ is torsion-free. For each subgroup $G<\operatorname{Mod}_{\Sigma}$, we set $G(m):=G \cap \operatorname{Mod}_{\Sigma}(m)$; it is a torsion-free finite-index normal subgroup of $G$. Ivanov made finer observations [13], §1.2, §1.6, on elements of $\operatorname{Mod}_{\Sigma}(m)$ and their reductions: every element $\sigma \in \operatorname{Mod}_{\Sigma}(m)$ is a neat element whose restriction to each component of $\Sigma$ is trivial or aperiodic; furthermore, for every reduction system $\mathcal{A}$ of $\sigma \in \operatorname{Mod}_{\Sigma}(m)$, the reduction $\rho_{\mathcal{A}}(\sigma)$ is a neat element in $\operatorname{Mod}_{\Sigma_{e}}$ whose restriction to each component of $\Sigma_{\mathscr{A}}$ is trivial or aperiodic. In particular, for every subgroup $G<\operatorname{Mod}_{\Sigma}, G(m) \triangleleft G$ is a neat finite-index normal subgroup of $G$.

Lemma 9 (Cf. [13], §7.11). Suppose $G<\operatorname{Mod}_{\Sigma}$ is adequately reduced. Then for every integer $m \geq 3$ the restriction of $G(m)$ to each component of $\Sigma$ is either (i) trivial or (ii) infinite and irreducible.

Proof. If $G<\operatorname{Mod}_{\Sigma}$ is adequately reduced, the finite-index normal subgroup $G(m)$ is also adequately reduced; hence, there exists a neat finite-index normal subgroup $G^{\prime} \triangleleft G(m)$ such that the restriction of $G^{\prime}$ to each component is either (i) trivial or (ii) infinite and irreducible. Since $G(m)$ is neat and its restriction to each component $\Sigma_{i}$ is either trivial or infinite, Lemma follows.

By Ivanov's classification theorem [13], §7.11, every subgroup $G<\operatorname{Mod}_{\Sigma}$ is either an adequately reduced subgroup or an infinite reducible subgroup with nonempty adequate reduction system. For an infinite reducible subgroup $G$, there is a canonical choice of non-empty reduction system [13], §7.2-7.4; it is indeed the unique minimal adequate reduction system for the subgroup $G$ [13], §7.18. We call such a system the canonical reduction system for $G$, and denote it by $\mathscr{C}_{G}$, or simply by $\mathcal{C}$ if the reference to $G$ is clear from the context.

4.2. Adequately reduced subgroups. We now give a description of adequately reduced subgroups based on Ivanov's theory. Some of the material is not entirely explicit in his exposition [13], Chap. 9; we shall extract and gather the relevant statements, and record a few consequences which we will utilize in the next section.

Connected surfaces. Suppose for now that $\Sigma$ is connected. Ivanov showed that $G(m) \triangleleft G$ contains a pair of independent pseudo-Anosov elements $\sigma$ and $\tau$ if $G(m)$ is irreducible and not infinite-cyclic [13], §5.12; high powers of $\sigma$ and $\tau$ then generates a non-abelian free subgroup of $G(m) \triangleleft G$ in the proof of the Tits alternative. 
Theorem 10 (Cf. [13], §5.12). Fix an integer $m \geq 3$. Let $\Sigma$ be a connected surface and let $G<\operatorname{Mod}_{\Sigma}$ be an adequately reduced subgroup. Then one of the following statements holds:

(0) $G(m) \triangleleft G$ is trivial, and $G$ is finite;

(1) $G(m) \triangleleft G$ is infinite-cyclic, and $G$ is virtually infinite-cyclic;

(2) $G(m) \triangleleft G$ contains an infinite number of pairwise independent pseudoAnosov elements.

Hence, if $G$ is neither finite nor virtually infinite-cyclic, then, for any finite collection $\varphi_{1}, \ldots, \varphi_{n} \in G(m) \triangleleft G$ of pseudo-Anosov elements, there exists a pseudo-Anosov element $\psi \in G(m) \triangleleft G$ such that $\psi$ and $\varphi_{j}$ are independent for all $j$.

Remark. Since $\Sigma$ is connected, it follows from Lemma 9 that $G(m)$ is either (i) trivial or (ii) infinite and irreducible. If $G(\mathrm{~m})$ is infinite and irreducible, $G(\mathrm{~m})$ contains a pseudo-Anosov element [13], §5.9; so, if $G(m)$ is infinite-cyclic, and hence irreducible, it follows that it is generated by a pseudo-Anosov element.

Proof. We assume $G(m)$ is neither trivial nor infinite-cyclic; by Lemma 9, $G(m)$ is irreducible. Hence, by Ivanov's result [13], §5.12, mentioned earlier, there exists a pair of independent pseudo-Anosov elements $\sigma, \tau \in G(m)$. For each $k \in \mathbb{Z}$, let $\psi_{k}:=\sigma^{k} \tau \sigma^{-k} \in G(m)$; it is a pseudo-Anosov element with $\operatorname{Fix}\left(\psi_{k}\right)=\sigma^{k}(\operatorname{Fix}(\tau))$. For $k \neq 0, \operatorname{Fix}\left(\sigma^{k}\right) \cap \operatorname{Fix}(\tau)=\operatorname{Fix}(\sigma) \cap \operatorname{Fix}(\tau)=\varnothing$ and hence $\operatorname{Fix}\left(\psi_{k}\right) \cap \operatorname{Fix}(\tau)=$ $\sigma^{k}(\operatorname{Fix}(\tau)) \cap \operatorname{Fix}(\tau)=\varnothing$. Then, more generally for $k \neq \ell, \operatorname{Fix}\left(\psi_{k}\right) \cap \operatorname{Fix}\left(\psi_{\ell}\right)=$ $\sigma^{\ell}\left(\sigma^{k-\ell}(\operatorname{Fix}(\tau))\right) \cap \sigma^{\ell}(\operatorname{Fix}(\tau))=\sigma^{\ell}\left(\operatorname{Fix}\left(\psi_{k-\ell}\right) \cap \operatorname{Fix}(\tau)\right)=\varnothing$; namely, $\Psi:=$ $\left\{\psi_{k} \mid k \in \mathbb{Z}\right\} \subset G(m)$ is a pairwise independent collection of pseudo-Anosov elements. Hence, for any finite collection $\varphi_{1}, \ldots, \varphi_{n} \in G(m)$ of pseudo-Anosov elements, there exists $\psi \in \Psi$ such that $\operatorname{Fix}\left(\varphi_{j}\right) \cap \operatorname{Fix}(\psi)=\varnothing$ for all $j$.

Disconnected surfaces. We now allow the surface $\Sigma=\bigsqcup_{i} \Sigma_{i}$ to be disconnected, where $\Sigma_{i}$ denotes each component. Ivanov showed that $G(m) \triangleleft G$ contains a pair of independent pseudo-Anosov elements $\sigma$ and $\tau$ if the restriction of $G(m)$ to each component $\Sigma_{i}$ is irreducible and not infinite-cyclic [13], §6.4. Generally, the restriction of $G(\mathrm{~m})$ to each component of $\Sigma$ falls into one of the three cases in Theorem 10. An adequately reduced group $G$ can be regarded as a hybrid of these three cases; this gives rise to a partition of $\Sigma$ into three subsurfaces.

Lemma 11 (Cf. [13], §9.10). Fix an integer $m \geq 3$, and let $G<\operatorname{Mod}_{\Sigma}$ be an adequately reduced subgroup. Consider a partition $\Sigma=\Sigma_{[0]} \sqcup \Sigma_{[1]} \sqcup \Sigma_{[2]}$ where each subsurface $\Sigma_{[\ell]}$ is defined as follows:

(0) the subsurface $\Sigma_{[0]}$ is the union of all components $\Sigma_{i}$ such that the restriction of $G(m)$ to $\Sigma_{i}$ is trivial;

(1) the subsurface $\Sigma_{[1]}$ is the union of all components $\Sigma_{i}$ such that the restriction of $G(m)$ to $\Sigma_{i}$ is infinite-cyclic; 
(2) the subsurface $\Sigma_{[2]}$ is the union of all components $\Sigma_{i}$ such that the restriction of $G(m)$ to $\Sigma_{i}$ is neither trivial nor infinite cyclic.

Then every element $\sigma \in G$ preserves this partition, i.e., $\sigma\left(\Sigma_{[\ell]}\right)=\Sigma_{[\ell]}$ for each $\ell$; hence, the restriction of $G$ to $\Sigma_{[\ell]}$ is well defined for each $\ell$.

The partition in Lemma 11 played no role in proving the Tits alternative for $\operatorname{Mod}_{\Sigma}$, while it serves as a critical step in proving the Margulis-Soifer theorem for Mod ${ }_{\Sigma}$ [13], Chap. 9. This partition is also essential in the proof of Theorem 1.

Proof. The proof we present here is essentially contained in [13], §9.10. Since $G(m) \triangleleft G$, the conjugate of an element $\tau \in G(m)$ by an element $\sigma \in G$ must again belong to $G(m)$; if $s$ and $t$ are homeomorphisms representing $\sigma$ and $\tau$ respectively, then $s \circ t \circ s^{-1}$ represents the element $\sigma \tau \sigma^{-1} \in G(m)$. For each component $\Sigma_{i}$, the restriction $\left.\tau\right|_{\Sigma_{i}}$ is represented by $\left.t\right|_{\Sigma_{i}}$, and the restriction $\left.\sigma \tau \sigma^{-1}\right|_{\sigma\left(\Sigma_{i}\right)}$ is represented by $s \circ\left(t \Sigma_{i}\right) \circ s^{-1}$. Thus, the conjugation by $\sigma$ defines a homomorphism from the restriction $\left.G(m)\right|_{\Sigma_{i}}$ to the restriction $\left.G(m)\right|_{\sigma\left(\Sigma_{i}\right)}$; it is clearly an isomorphism with the inverse being the conjugation by $\sigma^{-1}$. In particular, if the restrictions $\left.G(m)\right|_{\Sigma_{i}}$ and $\left.G(m)\right|_{\Sigma_{j}}$ are not isomorphic, e.g. if $\Sigma_{i}$ and $\Sigma_{j}$ belong to different parts in the partition $\Sigma=\Sigma_{[0]} \sqcup \Sigma_{[1]} \sqcup \Sigma_{[2]}$, then no element of $G$ can take $\Sigma_{i}$ to $\Sigma_{j}$.

Lemma 12. Fix an integer $m \geq 3$. Let $\Sigma=\Sigma_{[0]} \sqcup \Sigma_{[1]} \sqcup \Sigma_{[2]}$ be the partition in Lemma 11. For each $\ell$, let $G_{[\ell]}$ and $G(m)_{[\ell]}$ be the restrictions of $G$ and $G(m)$ to $\Sigma_{[\ell]}$ respectively, and set $G_{[\ell]}(m):=G_{[\ell]} \cap \operatorname{Mod}_{\Sigma_{[\ell]}}(m)$. Then $G(m)_{[\ell]}$ is a finite-index normal subgroup of $G_{[\ell]}(m)$.

Proof. By definition, $G_{[\ell]}(m)$ consists of all elements in $G_{[\ell]}$ which acts trivially on $H_{1}\left(\Sigma_{[\ell]} ; \mathbb{Z} / m \mathbb{Z}\right)<H_{1}(\Sigma ; \mathbb{Z} / m \mathbb{Z})$. Since $G(m)$ acts trivially on the entire $H_{1}(\Sigma ; \mathbb{Z} / m \mathbb{Z})$, its restriction $G(m)_{[\ell]}$ to $\Sigma_{[\ell]}$ acts trivially on $H_{1}\left(\Sigma_{[\ell]} ; \mathbb{Z} / m \mathbb{Z}\right) ;$ hence, $G(m)_{[\ell]}<G_{[\ell]}(m) \triangleleft G_{[\ell]}$. Since $G(m)$ is a finite-index normal subgroup of $G$, $G(m)_{[\ell]}$ must be a finite-index normal subgroup of $G_{[\ell]}$. Hence, we conclude that $G(m)_{[\ell]}$ must also be a finite-index normal subgroup of the intermediate subgroup $G_{[\ell]}(m)$.

Theorem 13 (Cf. [13], §9.10). Fix an integer $m \geq 3$, and let $G<\operatorname{Mod}_{\Sigma}$ be an adequately reduced subgroup. Let $\Sigma=\Sigma_{[0]} \sqcup \Sigma_{[1]} \sqcup \Sigma_{[2]}$ be the partition in Lemma 11 , and set $G_{[\ell]}$ and $G_{[\ell]}(m)$ as in Lemma 12. Then the following statements hold:

(0) $G_{[0]}(m) \triangleleft G_{[0]}$ is trivial, and $G_{[0]}$ is finite;

(1) $G_{[1]}(m) \triangleleft G_{[1]}$ is free-abelian, and $G_{[1]}$ is virtually free-abelian;

(2) $G_{[2]}(m) \triangleleft G_{[2]}$ contains an infinite number of pairwise independent pseudoAnosov elements. 
Hence, for any finite collection $\varphi_{1}, \ldots, \varphi_{n} \in G_{[2]}(m) \triangleleft G_{[2]}$ of pseudo-Anosov elements, there exists a pseudo-Anosov element $\psi \in G_{[2]}(m) \triangleleft G_{[2]}$ such that $\psi$ and $\varphi_{j}$ are independent for all $j$.

Proof. First, we consider $G(m)_{[0]}, G_{[0]}(m)$, and $G_{[0]} . G(m)_{[0]}$ is trivial by the choice of $\Sigma_{[0]}$ in Lemma 11; so, $G_{[0]}(m)$ is finite by Lemma 12, and hence $G_{[0]}$ is finite. We observe that $G_{[0]}(m)$ is actually trivial, since $G_{[0]}(m)$ is torsion-free.

Next, we consider $G(m)_{[1]}, G_{[1]}(m)$, and $G_{[1]}$. The restriction of $G(m)_{[1]}$ to each component $\Sigma_{i} \subset \Sigma_{[1]}$ is infinite-cyclic by the choice of $\Sigma_{[1]}$ in Lemma 11; hence, the restriction of $G_{[1]}(m)$ to $\Sigma_{i}$ must be virtually infinite-cyclic by Lemma 12 , and it is indeed infinite-cyclic by Theorem 10 . It follows that $G_{[1]}(m)$ must be free-abelian, and hence $G_{[1]}$ is virtually free-abelian.

Finally, we consider $G_{[2]}(m)$ and $G_{[2]}$. Note that it suffices to prove the statement (2) under the assumption $\Sigma=\Sigma_{[2]}$, and hence $G=G_{[2]}, G(m)=G_{[2]}(m)$. With this assumption, the restriction of $G(m)$ to each component $\Sigma_{i} \subset \Sigma_{[2]}=\Sigma$ is neither trivial nor infinite-cyclic by the choice of $\Sigma_{[2]}$ in Lemma 11, and is infinite and irreducible by Lemma 9. Hence, by Ivanov's result [13], §6.4, mentioned earlier, there exists a pair of independent pseudo-Anosov elements $\sigma, \tau \in G(m)$. As in the proof of Theorem 10, for each $k \in \mathbb{Z}$, let $\psi_{k}:=\sigma^{k} \tau \sigma^{-k} \in G(m)$; by the same argument, it follows that $\Psi:=\left\{\psi_{k} \mid k \in \mathbb{Z}\right\} \subset G(m)$ is a pairwise independent collection of pseudo-Anosov elements. Hence, for any finite collection $\varphi_{1}, \ldots, \varphi_{n} \in G(m)$ of pseudo-Anosov elements, there exists an element $\psi \in \Psi$ such that $\operatorname{Fix}\left(\varphi_{j}\right) \cap$ $\operatorname{Fix}(\psi)=\varnothing$ for all $j$.

\section{Girth alternative}

We now study the girth of subgroups of $\operatorname{Mod}_{\Sigma}$, for an orientable finite-type surface $\Sigma$ with $\partial \Sigma=\varnothing$. Our main result, the girth alternative, states that the dichotomy between the subgroups with infinite girth and the ones with finite girth coincides with the structural dichotomy of the Tits alternative, [12], [20].

Theorem 1. Let $\Sigma$ be an orientable finite-type surface with $\partial \Sigma=\varnothing$, and let $G$ be a finitely generated subgroup of $\operatorname{Mod}_{\Sigma}$. Then $G$ is either a non-cyclic group with infinite girth or a virtually free-abelian group; moreover, these alternatives are mutually exclusive.

Theorem 1 reduces to the special case, Theorem 14 below, where $\Sigma$ admits a complete hyperbolic metric. We first prove Theorem 1, assuming Theorem 14.

Theorem 14. Let $\Sigma$ be an orientable finite-type surface with $\partial \Sigma=\varnothing$, which admits a complete hyperbolic metric, and let $G$ be a finitely generated subgroup of $\mathrm{Mod}_{\Sigma}$. Then $G$ is either a non-cyclic group with infinite girth or a virtually free-abelian group; moreover, these alternatives are mutually exclusive. 
Proof of Theorem 1. Suppose for now that $\Sigma$ is a union of tori and hyperbolic components. Since the mapping class groups of tori and once-punctured tori are isomorphic, we may replace the copies of tori in $\Sigma$ with the same number of copies of once-punctured tori. In turn, we now realize $G$ as a subgroup of the mapping class group of a hyperbolic surface; Theorem 1 follows from Theorem 14 as desired.

For the general case, let $\Sigma=\Sigma^{+} \sqcup \Sigma^{-}$where $\Sigma^{+}$is the union of spheres with at most two punctures and $\Sigma^{-}$is the union of tori and hyperbolic components. Let $G^{-}$ be the restriction of $G$ to $\Sigma^{-}$; we have shown above that $G^{-}$is either a non-cyclic group with infinite girth or a virtually free-abelian group. Note that the restriction $G^{-}$ of the subgroup $G$ to $\Sigma^{-}$is the image of the restriction homomorphism $G \rightarrow \operatorname{Mod}_{\Sigma^{-}}$; hence, if $G^{-}$is a non-cyclic group with infinite girth, then so is $G$ by Proposition 5. So, we assume that $G^{-}$is virtually free-abelian and let $A<G^{-}$be a finite-index free-abelian subgroup.

The mapping class group is trivial for the sphere and the sphere with one puncture, and is isomorphic to $\mathbb{Z} / 2 \mathbb{Z}$ for the sphere with two punctures; hence, $\operatorname{Mod}_{\Sigma^{+}}$is finite. It follows that the kernel $K \triangleleft G$ of the restriction homomorphism $G \rightarrow \operatorname{Mod}_{\Sigma^{+}}$is a finite-index normal subgroup of $G$. Let $K^{-} \triangleleft G^{-}$be the restriction of $K$ to $\Sigma^{-}$. Then $K^{-} \cap A$ is a finite-index free-abelian subgroup of $K^{-}$. Now, observe that $K$ and $K^{-}$are actually isomorphic since $K$ acts trivially on $\Sigma^{+}$. Hence, $K$ contains a finite-index free-abelian subgroup $H<K$, corresponding to $K^{-} \cap A<K^{-}$. Since $K$ is a finite-index subgroup of $G$, it follows that $H<K<G$ is a finite-index free-abelian subgroup of $G$; in other words, $G$ is virtually free-abelian as desired.

We prove Theorem 14 in the rest of this section. In \$5.1, we treat adequately reduced subgroups with Propositions 15 for connected surfaces and with Proposition 17 for disconnected surfaces; in §5.2, we treat reducible subgroups with Proposition 19. In Proposition 17 and 19, we apply Proposition 2 to the image of the surjections, suitably chosen by the results from $\S 4$, when the given subgroup is not virtually free-abelian.

5.1. Adequately reduced subgroups. We first consider an adequately reduced subgroup $G<\operatorname{Mod}_{\Sigma}$, where $\Sigma$ is a connected surface.

Proposition 15. Let $\Sigma$ be an orientable connected finite-type surface with $\partial \Sigma=\varnothing$, which admits a complete hyperbolic metric, and let $G<\operatorname{Mod}_{\Sigma}$ be an adequately reduced subgroup. Then $G$ is either a non-cyclic group with infinite girth, a virtually infinite-cyclic group, or a finite group; these alternatives are mutually exclusive.

Proof. Choose an integer $m \geq 3$. Suppose $G$ is an adequately reduced subgroup of $\operatorname{Mod}_{\Sigma}$, and let $S=\left\{\gamma_{1}, \ldots, \gamma_{n}\right\}$ be a generating set of $G$. We assume that $G$ is neither virtually infinite-cyclic nor finite, and aim to show that it has infinite girth.

In this case, the statement (2) of Theorem 10 is satisfied. We know that there is a pseudo-Anosov element $\sigma \in G(m) \triangleleft G$. For each $1 \leq j \leq n$ and $\varepsilon=$ 
\pm 1 , the conjugate $\gamma_{j}^{\varepsilon} \sigma \gamma_{j}^{-\varepsilon} \in G(m) \triangleleft G$ is again a pseudo-Anosov element with $\operatorname{Fix}\left(\gamma_{j}^{\varepsilon} \sigma \gamma_{j}^{-\varepsilon}\right)=\gamma_{j}^{\varepsilon}(\operatorname{Fix}(\sigma))$. Hence, applying the statement (2) of Theorem 10 to the collection $\{\sigma\} \cup\left\{\gamma_{j}^{\varepsilon} \sigma \gamma_{j}^{-\varepsilon} \mid 1 \leq j \leq n, \varepsilon= \pm 1\right\}$, we see that there is another pseudo-Anosov element $\tau \in G(m) \triangleleft G$ such that $\operatorname{Fix}(\tau) \cap \operatorname{Fix}(\sigma)=\varnothing$ and $\operatorname{Fix}(\tau) \cap \operatorname{Fix}\left(\gamma_{j}^{\varepsilon} \sigma \gamma_{j}^{-\varepsilon}\right)=\varnothing$ for all $1 \leq j \leq n$ and $\varepsilon= \pm 1$.

Note that, if $U_{\sigma}$ is a neighborhood of $\operatorname{Fix}(\sigma)$, then $\gamma_{j}^{\varepsilon}\left(U_{\sigma}\right)$ is a neighborhood of $\operatorname{Fix}\left(\gamma_{j}^{\varepsilon} \sigma \gamma_{j}^{-\varepsilon}\right)$ for each $1 \leq j \leq n$ and $\varepsilon= \pm 1$. It then follows that there are small enough neighborhoods $U_{\sigma} \supset \operatorname{Fix}(\sigma)$ and $U_{\tau} \supset \operatorname{Fix}(\tau)$ such that

$$
U_{\sigma} \cap U_{\tau}=\varnothing \quad \text { and } \quad U_{\tau} \cap \gamma_{j}^{\varepsilon}\left(U_{\sigma}\right)=\varnothing
$$

for each $1 \leq j \leq n$ and $\varepsilon= \pm 1$, or equivalently

$$
U_{\sigma} \cap U_{\tau}=\varnothing \quad \text { and } \quad \gamma_{j}^{\varepsilon}\left(U_{\tau}\right) \cap U_{\sigma}=\varnothing
$$

for each $1 \leq j \leq n$ and $\varepsilon=\mp 1$. Now, since $\sigma$ and $\tau$ are pseudo-Anosov elements, we can take high enough powers $\tilde{\sigma}:=\sigma^{N}$ and $\tilde{\tau}:=\tau^{N}$ such that

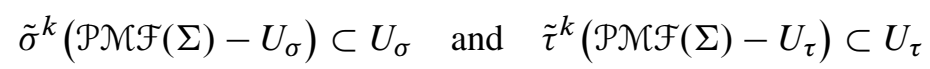

for all non-zero integer $k$. In particular, we have

$$
\tilde{\sigma}^{k}\left(U_{\tau} \cup \bigcup_{\varepsilon= \pm 1} \bigcup_{j=1}^{n} \gamma_{j}^{\varepsilon}\left(U_{\tau}\right)\right) \subset U_{\sigma} \quad \text { and } \quad \tilde{\tau}^{k}\left(U_{\sigma} \cup \bigcup_{\varepsilon= \pm 1} \bigcup_{j=1}^{n} \gamma_{j}^{\varepsilon}\left(U_{\sigma}\right)\right) \subset U_{\tau}
$$

for all non-zero integer $k$. Applying Proposition 2 to $\tilde{\sigma}, \tilde{\tau}, U_{\sigma}, U_{\tau}$, and

$$
x \in \mathcal{P} \mathcal{M} F(\Sigma)-\left(\left(U_{\sigma} \cup U_{\tau}\right) \cup \bigcup_{\varepsilon= \pm 1} \bigcup_{j=1}^{n} \gamma_{j}^{\varepsilon}\left(U_{\sigma} \cup U_{\tau}\right)\right)
$$

we conclude that $G$ must be a non-cyclic group with infinite girth.

We now consider an adequately reduced subgroup $G<\operatorname{Mod}_{\Sigma}$ where $\Sigma$ is disconnected. The strategy is to take the partition $\Sigma=\Sigma_{[0]} \sqcup \Sigma_{[1]} \sqcup \Sigma_{[2]}$ from Lemma 11, and restrict the group $G$ to $\Sigma_{[2]}$ when $\Sigma_{[2]} \neq \varnothing$; the non-emptiness of $\Sigma_{[2]}$ then guarantees the infinite girth. When $\Sigma_{[2]}$ is empty, the following lemma from the proof of the Tits alternative shows that $G$ is virtually free-abelian.

Lemma 16 (Cf. [13], §8.7). Let $m \geq 3$ be an integer, and let $G$ be an adequately reduced group. Then $G$ is virtually free-abelian if and only if the restriction of $G(m)$ to each component of $\Sigma$ is either trivial or infinite-cyclic, i.e., $\Sigma_{[2]}=\varnothing$ in the partition from Lemma 11.

Proposition 17. Let $\Sigma$ be an orientable finite-type surface with $\partial \Sigma=\varnothing$, which admits a complete hyperbolic metric, and let $G<\operatorname{Mod}_{\Sigma}$ be an adequately reduced subgroup. Then $G$ is either a non-cyclic group with infinite girth or a virtually free-abelian group; these alternatives are mutually exclusive. 
Proof. Consider the decomposition $\Sigma=\Sigma_{[0]} \sqcup \Sigma_{[1]} \sqcup \Sigma_{[2]}$ in Lemma 11. If $\Sigma_{[2]}=$ $\varnothing$, then $G$ is virtually free-abelian by Lemma 16 . Hence, we may assume that $\Sigma_{[2]} \neq \varnothing$. Note that the restriction $G_{[2]}$ of the subgroup $G$ to $\Sigma_{[2]}$ is the image of the restriction homomorphism $G \rightarrow \operatorname{Mod}_{\Sigma_{[2]}}$; hence, if the restriction $G_{[2]}$ is a non-cyclic group with infinite girth, then so is $G$ by Proposition 5; it remains to show that $G_{[2]}$ is indeed a non-cyclic group with infinite girth.

For this, we may as well assume that $\Sigma=\Sigma_{[2]}$ and hence $G=G_{[2]}$. Choose $m \geq$ 3. Let $S=\left\{\gamma_{1}, \ldots, \gamma_{n}\right\}$ be a generating set of $G$. By Theorem 13 , we know that there is a pseudo-Anosov element $\sigma \in G(m) \triangleleft G$, and there is another pseudo-Anosov element $\tau \in G(m) \triangleleft G$ such that $\operatorname{Fix}(\tau) \cap \operatorname{Fix}(\sigma)=\varnothing$ and $\operatorname{Fix}(\tau) \cap \operatorname{Fix}\left(\gamma_{j}^{\varepsilon} \sigma \gamma_{j}^{-\varepsilon}\right)$ for each $1 \leq j \leq n$ and $\varepsilon= \pm 1$. Here, the conjugates $\gamma_{j}^{\varepsilon} \sigma \gamma_{j}^{-\varepsilon}$ are pseudo-Anosov elements with $\operatorname{Fix}\left(\gamma_{j}^{\varepsilon} \sigma \gamma_{j}^{-\varepsilon}\right)=\gamma_{j}^{\varepsilon}(\operatorname{Fix}(\sigma))$ in $\mathcal{P M}_{\mathcal{M}}^{\sharp}(\Sigma)$. From the discussion in $\S 3.2$, we know that the action of pseudo-Anosov elements in $\operatorname{Mod}_{\Sigma}$ on $\mathcal{P \mathcal { M }} \mathcal{F}^{\sharp}(\Sigma)$ exhibits the north-south dynamics; hence, by the arguments identical to the proof of Proposition 15 - with the space $\mathcal{P \mathcal { N F }} \mathcal{F}(\Sigma)$ replaced by $\mathcal{P} \mathcal{N} \mathcal{F}^{\sharp}(\Sigma)$ - we see that $G$ is a non-cyclic group with infinite girth by Proposition 2.

5.2. Reducible subgroups. We now consider a reducible group $G<\operatorname{Mod}_{\Sigma}$. To prove the girth alternative, we take the canonical reduction $\rho_{e}(G)<\operatorname{Mod}_{\Sigma_{e}}$, for which the girth alternative holds by Proposition 17. The following lemma, extracted from the proof of the Tits alternative [13], §8.9, characterizes virtually free-abelian subgroups $G$ in terms of its reduction $\rho e(G)$.

Lemma 18 (Cf. [13], §8.9). A reducible subgroup $G<\operatorname{Mod}_{\Sigma}$ is virtually freeabelian if and only if the canonical reduction $\rho e(G)$ is virtually free-abelian.

Proposition 19. Let $\Sigma$ be an orientable finite-type surface with $\partial \Sigma=\varnothing$, which admits a complete hyperbolic metric, and let $G<\operatorname{Mod}_{\Sigma}$ be a reducible subgroup. Then $G$ is either a non-cyclic group with infinite girth or a virtually free-abelian group; these alternatives are mutually exclusive.

Proof. The canonical reduction $\rho_{e}(G)$ of $G$ is adequately reduced, and hence it is either a non-cyclic group with infinite girth or a virtually free-abelian group by Proposition 17. Recall that the canonical reduction $\rho_{e}(G)$ is indeed the image of the reduction homomorphism $\rho_{e}: G \rightarrow \operatorname{Mod}_{\Sigma_{e}}$; hence, if $\rho_{e}(G)$ is a non-cyclic group with infinite girth, so is $G$ by Proposition 5. If $\rho e(G)$ is virtually free-abelian, so is $G$ by Lemma 18 . 


\section{References}

[1] S. I. Adyan, Random walks on free periodic groups. Izv. Akad. Nauk SSSR Ser. Mat. 46 (1982), 1139-1149; English transl. Math. USSR-Izv. 21 (1983), 425-434.Zbl 0512.60012 MR 0682486

[2] A. Akhmedov, On the girth of finitely generated groups. J. Algebra 268 (2003), 198-208. Zbl 1037.20030 MR 2004484

[3] A. Akhmedov, The girth of groups satisfying Tits alternative. J. Algebra 287 (2005), 275-282. Zbl 1087.20023 MR 2134144

[4] A. Akhmedov, Girth alternative for subgroups of $P L_{o}(I)$. Preprint 2011. arXiv: 1105.4908

[5] A. Akhmedov, M. Stein, and J. Taback, Free limits of Thompson's group F. Geom. Dedicata 155 (2011), 163-176. Zbl 1275.20043 MR 2863899

[6] J. S. Birman, A. Lubotzky, and J. McCarthy, Abelian and solvable subgroups of the mapping class groups. Duke Math. J. 50 (1983), 1107-1120. Zbl 0551.57004 MR 726319

[7] J. Bourgain and A. Gamburd, Uniform expansion bounds for Cayley graphs of $\operatorname{SL}_{2}\left(\mathbb{F}_{p}\right)$. Ann. of Math. (2) 167 (2008), 625-642. Zbl 1216.20042 MR 2415383

[8] J. Bourgain and P. P. Varjú, Expansion in $\mathrm{SL}_{d}(\mathbb{Z} / q \mathbb{Z}), q$ arbitrary. Invent. Math. 188 (2012), 151-173. Zbl 1247.20052 MR 2897695

[9] M. G. Brin, The free group of rank 2 is a limit of Thompson's group F. Groups Geom. Dyn. 4 (2010), 433-454. Zbl 1248.20046 MR 2653970

[10] M. G. Brin and C. C. Squier, Groups of piecewise linear homeomorphisms of the real line. Invent. Math. 79 (1985), 485-498. Zbl 0563.57022 MR 782231

[11] A. Fathi and F. Laudenbach and V. Peénaru, Thurston's work on surfaces. Math. Notes 48, Princeton University Press, Princeton, NJ, 2012. Zbl 1244.57005 MR

[12] N. V. Ivanov, Algebraic properties of the Teichmüller modular group. Dokl. Akad. Nauk SSSR 275 (1984), 786-789; English transl. Soviet Math. Dokl. 29 (1984), 288--291. Zbl 0586.20026 MR 745513

[13] N. V. Ivanov, Subgroups of Teichmüller modular groups. Transl. Math. Monographs 115, Amer. Math. Soc., Providence, RI, 1992. Zbl 0776.57001 MR 1195787

[14] G. Keller, Amenable groups and varieties of groups. Illinois J. Math. 16 (1972), 257-269. Zbl 0231.22006 MR 0296141

[15] F. Klein, Neue Beiträge zur Riemann'schen Functionentheorie. Math. Ann. 21 (1883), 141-218. JFM 15.0351.01

[16] A. Lubotzky, R. Phillips, and P. Sarnak, Ramanujan graphs. Combinatorica 8 (1988), 261-277. Zbl 0661.05035 MR 963118

[17] J. Mangahas, Uniform uniform exponential growth of subgroups of the mapping class group. Geom. Funct. Anal. 19 (2010), 1468-1480. Zbl MR 2585580

[18] G. A. Margulis, Explicit constructions of graphs without short cycles and low density codes. Combinatorica 2 (1982), 71-78. Zbl 0492.05044 MR 671147

[19] G. A. Margulis and G. A. Soifer, Maximal subgroups of infinite index in finitely generated linear groups. J. Algebra 69 (1981), 1-23. Zbl 0457.20046 MR 613853 
[20] J. McCarthy, A “Tits-alternative" for subgroups of surface mapping class groups. Trans. Amer. Math. Soc. 291 (1985), 583-612. Zbl 0579.57006 MR 800253

[21] K. Nakamura, Some results in topology and group theory. Ph.D. Thesis, University of California, Davis 2008.

[22] J. Nielsen, Abbildungsklassen endlicher Ordnung. Acta Math. 75 (1943), 23-115. Zbl 0027.26601 MR 0013306

[23] A. Ju. Ol'šanskiĭ, An infinite group with subgroups of prime orders. Izv. Akad. Nauk SSSR Ser. Mat. 44 (1980), 309-321; English transl. Math. USSR-Izv. 16 (1981), 279-289. Zbl 0475.20025 MR 571100

[24] A. Yu. Ol'shanskii, On the problem of the existence of an invariant mean on a group. Uspekhi Mat. Nauk 35 (1980), no. 4, 199-200; English transl. Russian Math. Surveys 35 (1980), no. 4, 180-181. Zbl 0465.20030 MR 586204

[25] A. Yu. Ol'shanskii, Geometry of defining relations in groups. Math Appl. (Soviet Series) 70, Kluwer Academic Publishers, Dordrecht 1991. Zbl 0732.20019 MR 1191619

[26] A. Yu. Ol'shanskii and M. V. Sapir, Non-amenable finitely presented torsion-by-cyclic groups. Publ. Math. Inst. Hautes Études Sci. 96 (2002), 43-169. Zbl 1050.20019 MR 1985031

[27] S. Schleimer, On the girth of groups. Preprint 2000. http://homepages.warwick.ac.uk/ masgar/Maths/girth.pdf

[28] J.-P. Serre, Rigidité du foncteur de Jacobi d'échelon $n \geq 3$. In Séminaire Henri Cartan 13, no. 2, 1960-1961, Exp. 17, Appendix to A. Grothendieck, Techniques de construction en géométrie analytique, X. Construction de l'espace de Teichmüller, Secrétariat mathématique, Paris 1960-1961.

http://archive.numdam.org/article/SHC_1960-1961_13_2_A4_0.pdf

[29] W. P. Thurston, On the geometry and dynamics of diffeomorphisms of surfaces. Bull. Amer. Math. Soc. (N.S.) 19 (1988), 417-431. Zbl 0674.57008 MR 956596

[30] J. Tits, Free subgroups in linear groups. J. Algebra 20 (1972), 250-270. Zbl 0236.20032 MR 0286898

[31] S. Yamagata, The girth of convergence groups and mapping class groups. Osaka J. Math. 48 (2011), 233-249. Zbl 1270.20033 MR 2802600

Received September 1, 2010; revised December 27, 2012

K. Nakamura, Mathematical Sciences Building, One Shields Ave., University of California, Davis, CA 95616, U.S.A.

E-mail: knakamura@math.ucdavis.edu 